\title{
Dimensionality reduction applied to logical judgments
}

\author{
Author: \\ Cheng Yuan yuan（中文: 程园园） \\ Address for correspondence: \\ E-mail:henuhh@outlook.com
}

\begin{abstract}
:
Purpose: To study the effect of the application of the dimensionality reduction in logical judgment (or logical reasoning, logical inference) programs. Methods: Use enumeration and dimensionality reduction methods to solve logical judgment problems. The effect of the two methods is illustrated in the form of a case study. Results: For logical judgmentproblems, using enumeration method to find the best answer is a comprehensive and fundamental method, but the disadvantage is that it is computationally intensive and computationally inefficient. Compared with the ideas of parallel treatment of known conditions by enumeration method, the application of dimensionality reduction thinking was built on the basis of fully mining information for feature extraction and feature selection. Conclusions: The dimensionality reduction method was applied to the logical judgment problems, and on the basis of fully mining information, the dimensionality reduction principle of statistics were applied to stratify and merge variables with the same or similar characteristics to achieve the purpose of streamlining variables, simplifying logical judgment steps, reducing computation and improving algorithm efficiency.
\end{abstract}

Keywords: dimensionality reduction; logic judgment; enumeration method

The enumeration method was applied to logical judgment problems to find the best answer by treating known conditions in parallel and filtering all possible combinations, which is a basic and comprehensive algorithm. The disadvantage is that it is computationally intensive and not very efficient. The dimensionality reduction method was applied to the logical judgment problems, and 
on the basis of fully mining information, the dimensionality reduction principle of statistics were applied to stratify and merge variables with the same or similar characteristics to achieve the purpose of streamlining variables, simplifying logical judgment steps, reducing computation and improving algorithm efficiency.

The application of the two methods was illustrated in the form of a case study respectively.

\section{The first case was shown below .}

In a school, a student did a good deed without keeping his name, and after the letter of commendation was sent to the school, the principal approached the four students concerned and asked, "Who did the good deed?" The four students' answers were: A said: not me; B said: C; C said: D; D said: C nonsense. Three of the students told the truth, and one of them told a lie. Based on this information, find out who is the student who did the good deed? ${ }^{[1]}$

\section{Enumeration Method ${ }^{[1]}$.}

Ideas: Use a computer language to create looping statements that make logical judgments about all possible combinations and find the ones that also satisfy the known conditions.

Methods: Assume that $\mathrm{X}$ is the person who did the good deed, and the value of $\mathrm{X}$ is $\mathrm{A}, \mathrm{B}, \mathrm{C}$ and $\mathrm{D}$ in order.

First, let $\mathrm{X}=\mathrm{A}$. Based on the known conditions, make a logical judgment as follows.

To determine whether A was telling the truth.

To determine whether B was telling the truth.

To determine whether $\mathrm{C}$ was telling the truth.

To determine whether $\mathrm{D}$ was telling the truth.

Next, make $\mathrm{X}=\mathrm{B}, \mathrm{X}=\mathrm{C}$, and $\mathrm{X}=\mathrm{D}$ in turn, and perform the same logical judgments to find out if the words of three students were true at the same time when the 4-fold cycle ends. If the result was true, the student whose $\mathrm{X}$ value corresponds to the one who did the good deed. The results of the 4-loop logical judgment were shown in Table 1. The programming codes were omitted. 
Table 1: The results of the 4-loop logical judgment

\begin{tabular}{ccccc}
\hline & X & & & \\
& A & B & C & D \\
A & false & true & true & true \\
B & false & false & true & false \\
C & false & false & false & true \\
D & true & true & true & false \\
judgments that turn out to & & & & \\
be true & 1 & 2 & 3 & 2 \\
\end{tabular}

\section{Dimensionality reduction method.}

Ideas: The information were fully mined to find out the variables suitable for stratification and merging to achieve dimensionality reduction, and to simplify the calculation steps and computation of the logical judgment problems.

Methods.

Let variables A, B, C, and D denote the words spoken by students A, B, C, and D, respectively. Under the premise that B and D were not mutually exclusive, B and D were stratified (or classified) and combined. It was clear from the title that if B was true, then C was false and vice versa; if $\mathrm{C}$ was true, then $\mathrm{D}$ was false and vice versa. That was, $\mathrm{B}$ and $\mathrm{C}$ were mutually exclusive, and C and D were mutually exclusive. So, can B and D be mutually exclusive? The answer was: NO.

It can be proved that.

It was known that $\mathrm{B}$ and $\mathrm{C}$ were mutually exclusive and $\mathrm{C}$ and $\mathrm{D}$ were mutually exclusive. If $\mathrm{B}$ and $\mathrm{D}$ were mutually exclusive, then $\mathrm{C}$ and $\mathrm{D}$ were not mutually exclusive, which contradicts the known fact that $\mathrm{C}$ and $\mathrm{D}$ were mutually exclusive, therefore, $\mathrm{B}$ and $\mathrm{D}$ were not mutually 
exclusive. Thus, B and D were stratified (or classified) and merged to reduce the dimensionality of the logical judgment problems.

The ideas and methods of dimensionality reduction analysis of logical judgment were represented in Figure 1.

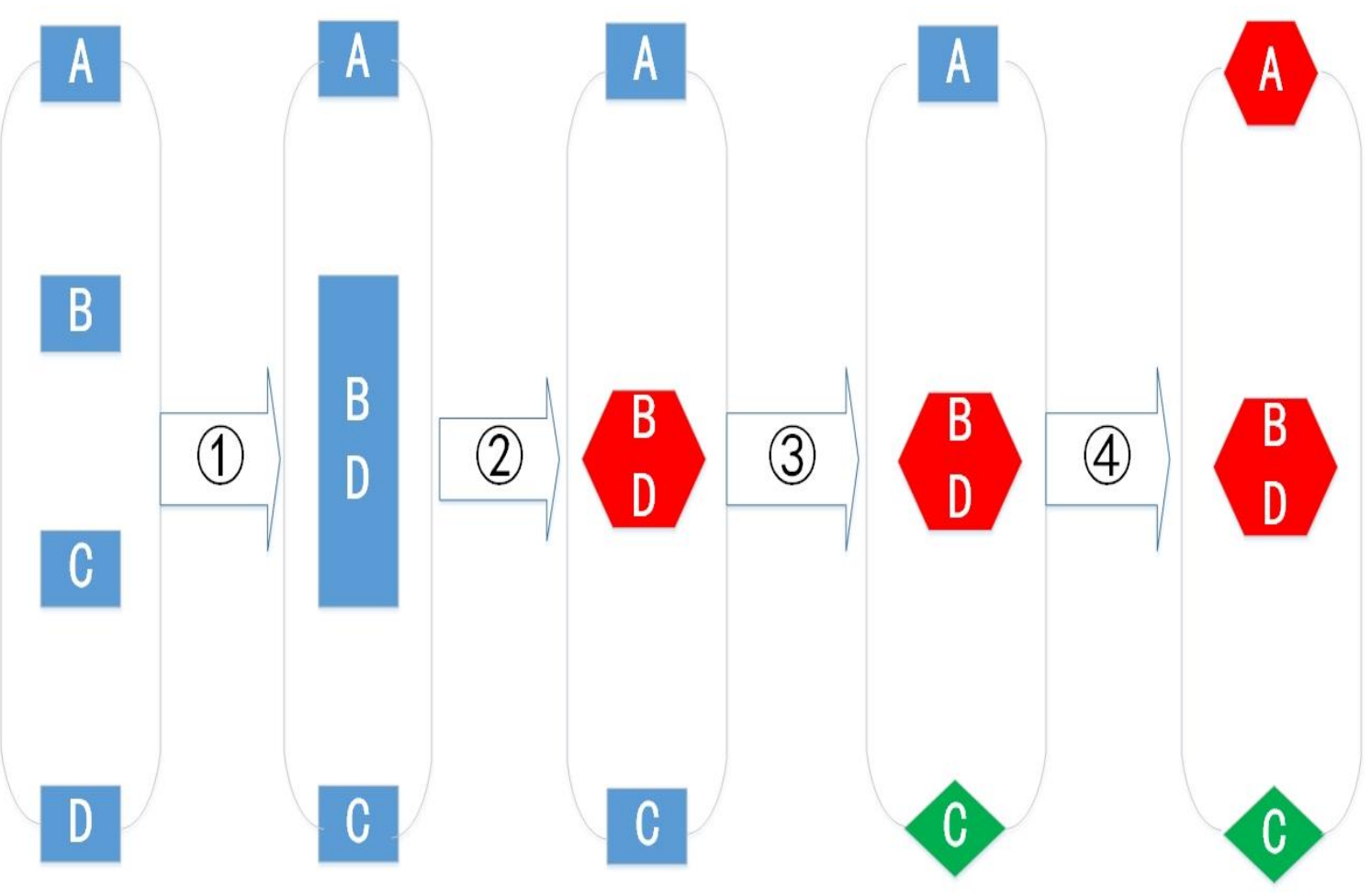

Fig. 1 Dimensionality reduction analysis ideas and methods of logical judgment diagram

\section{Steps:}

(1) Classify B and D into the same layer (or class), because B and D can be true or false at the same time; (2) Since only one of the four people said something false, B and D cannot be false at the same time, but only true at the same time; (3) Since B was true, C was false; (4) Further (same as (2)) it can be deduced that A was true. Thus, we can find out that the student who did a good deed was: C.

As can be seen, stratification and merging variables achieve dimensionality reduction, simplifying the logical judgment problems between four people into a logical judgment problems between three people or even two people, where the correct answer has been found in step (3) and 
only further verification was required in step (4). In this way, the calculation steps and the calculation volume of the logical judgment problem were greatly simplified, while the use of logical thinking skills were emphasized.

\section{The second case.}

The second case was that a local criminal investigation brigade analyzed a suspicious case involving six suspects.

(1) At least one of A and B committed the crime.

(2) At least two of A, E and F were involved in the crime.

(3) A and D were unlikely to be co-perpetrators.

(4) B and C either committed the crime at the same time or were unrelated to the case.

(5) one and only one of C and D committed the crime.

(6) If D was not involved in the crime, then $\mathrm{E}$ was not likely to be involved in the crime either.

Try to design algorithms to find out the perpetrators of the crime ${ }^{[1]}$.

Each of the six suspects had the possibility of committing or not committing a crime. The enumeration method required six cycles to find the eligible combinations from $2^{6}$ combinations, and the logical judgment methods and program codes of the enumeration method were omitted.

\section{Dimensionality reduction method.}

Let the variables a, b, c, d, e, and f denote suspects A, B, C, D, E, and F, respectively. Let 1 and 0 denote perpetrators and non-perpetrators, respectively.

It was known that $b$ and $c$ either work at the same time or were unrelated to the case.

Thus, $\mathrm{b}$ and $\mathrm{c}$ were classified into the same layer (or class). When $\mathrm{d}$ can be ruled out as a suspect, $\mathrm{d}$ was classified as the same layer (or class) as e. It was also known that $\mathrm{b}$ and $\mathrm{c}$ were associated with $\mathrm{d}$ and a, which in turn were associated from $\mathrm{d}$ to $\mathrm{e}$ and from e to f. 
Assuming that $\mathrm{b}=0$ and $\mathrm{b}$ and $\mathrm{c}$ were divided into the same layer (or class), then $\mathrm{c}=0$. $\mathrm{By}$ the condition (5) associated with $\mathrm{c}, \mathrm{d}=1$, and by condition (3) associated with $\mathrm{d}, \mathrm{a}=0$. Both a and b were 0 , which contradicts condition (1), and therefore, $b=0$ did not hold.

Thus, let $\mathrm{b}=1$ and $\mathrm{b}$ and $\mathrm{c}$ were in the same layer (or class), then $\mathrm{c}=1$. From condition (5), which was related to $c$, it followed that $d=0$. When $d$ was ruled out as a suspect, and $d$ was in the same layer as e, then $\mathrm{e}=0$, and from the condition (2) related to e, we knew that $\mathrm{a}=1$ and $\mathrm{f}=1$.

Therefore, when A, B, C, and F were the perpetrators, and D and E were excluded from suspicion of the crime, the above conditions were satisfied at the same time.

Dimensionality reduction was often achieved in statistics by extracting features of variables and merging related variables, using linear dimensionality reduction techniques and nonlinear dimensionality reduction techniques, including that principal component analysis (PCA ), singular value decomposition (SVD), latent semantic analysis (LSA), locality preserving projectionS (LPP), independent component analysis (ICA), linear discriminant analysis (LDA), projection pursuit(PP), and kernel principal component analysis (KPCA), multidimensional scaling (MDS), isometric mapping (ISOMAP), locally linear embedding (LLE), self-organizing map(SOM), learning vector quantization (LVQ), t-stochastic neighbor embedding (t-SNE), etc ${ }^{[2-16]}$, to achieve the purpose of reducing the covariance between variables, rationalizing the interpretation of statistical models, reducing data redundancy and improving the efficiency of the algorithms.

Compared with the ideas of parallel treatment of known conditions by enumeration method, the application of dimensionality reduction thinking was built on the basis of fully mining information for feature extraction and feature selection, which paid more attention to logical thinking ability and simplifies the steps of logical judgment, which was beneficial to the improvement and enhancement of algorithms, and had a facilitating effect in big data applications, especially for machine learning.

In addition, logical judgment with the thinking of dimensionality reduction, starting from merging variables, can find the entry point of logical judgment problems more quickly, and with the principle of sequential analysis ${ }^{[17]}$, it can further improve the efficiency of problem solving. 


\section{References.}

[1] Wu Wenhu. How to teach "Programming Fundamentals"[J]. China University

Teaching,2011(12):10-12.

[2] Harold Hotelling. Analysis of a complex of statistical variables into principal components.[J].

Journal of Educational Psychology,1933,24(6):pp. 417-441 and 498-520.doi:10.1037/H0070888.

[3] K. Pearson. LIII. On lines and planes of closest fit to systems of points in space[J].

Philosophical Magazine Series 6,1901,2(11): pp. 559-572.doi:10.1080/14786440109462720.

[4] Van Der Maaten L, Postma E, Van den Herik J. Dimensionality reduction: a comparative[J]. J Mach Learn Res, 2009, 10(66-71): 13.

[5] Ayesha S , Hanif M K, Talib R. Overview and comparative study of dimensionality reduction techniques for high dimensional data[J]. Information Fusion, 2020, 59:44-58.

[6] Ray, P., Reddy, S.S. \& Banerjee, T. Various dimension reduction techniques for high dimensional data analysis: a review[J]. Artif Intell Rev 54, 3473-3515

(2021).https://doi.org/10.1007/s10462-020-09928-0

[7] Cunningham John P, Ghahramani Zoubin. Linear Dimensionality Reduction: Survey, Insights, and Generalizations[J]. Journal of Machine Learning Research. 2015,16(1):2859-2900.

[8] Tenenbaum J B, De Silva V, Langford J C. A global geometric framework for nonlinear dimensionality reduction[J]. science, 2000, 290(5500): 2319-2323.

[9] Roweis S T, Saul L K. Nonlinear dimensionality reduction by locally linear embedding[J]. science, 2000, 290(5500): 2323-2326.

[10] De Silva V, Tenenbaum J B. Global versus local methods in nonlinear dimensionality reduction[C]//NIPS. 2002, 15: 705-712.

[11] Belkin M, Niyogi P. Laplacian eigenmaps for dimensionality reduction and data representation[J]. Neural computation, 2003, 15(6): 1373-1396.

[12] Bingham E, Mannila H. Random projection in dimensionality reduction: applications to image and text data[C]/Proceedings of the seventh ACM SIGKDD international conference on Knowledge discovery and data mining. 2001: 245-250.

[13] Hasan B M S, Abdulazeez A M. A Review of Principal Component Analysis Algorithm for Dimensionality Reduction[J]. Journal of Soft Computing and Data Mining, 2021, 2(1): 20-30. 
[14] Anowar F, Sadaoui S, Selim B. Conceptual and empirical comparison of dimensionality reduction algorithms (PCA, KPCA, LDA, MDS, SVD, LLE, ISOMAP, LE, ICA, t-SNE)[J]. Computer Science Review, 2021, 40: 100378.

[15] Reddy G T, Reddy M P K, Lakshmanna K, et al. Analysis of dimensionality reduction techniques on big data[J]. IEEE Access, 2020, 8: 54776-54788.

[16] Zebari R, Abdulazeez A, Zeebaree D, et al. A comprehensive review of dimensionality reduction techniques for feature selection and feature extraction[J]. Journal of Applied Science and Technology Trends, 2020, 1(2): 56-70.

[17] Abraham Wald . Sequential tests of statistical hypothesis[J]. Annals of Mathematical Statistics, 1945, 16(2): 117-186. 


\section{Competing Interest Statement}

The authors declare no competing interests.

\section{Funding Statement}

There is no external funding, and there is no conflict of interest.

\section{Author Declaration}

All relevant ethical guidelines have been followed; any necessary IRB and/or ethics committee approvals have been obtained, and details of the IRB/oversight body are included in the manuscript.

Yes

All necessary patient/participant consent was obtained, and the appropriate institutional forms were archived.

Yes

I understand that all clinical trials and any other prospective interventional studies must be registered with an ICMJE-approved registry, such as ClinicalTrials.gov. I confirm that any such study reported in the manuscript has been registered and the trial registration ID is provided (note: if posting a prospective study registered retrospectively. Please provide a statement in the trial ID field explaining why the study was not registered in advance).

Yes

I have followed all appropriate research reporting guidelines and uploaded the relevant EQUATOR Network research reporting checklist(s) and other pertinent materials as supplementary files, if applicable.

Yes

Data Availability Statement:

All data, models, and code generated or used during the study appear in the submitted article. 